\title{
Complement inhibitory proteins expression in placentas of thrombophilic women
}

\author{
Przemysław Krzysztof Wirstlein ${ }^{1}$, Piotr Jasiński ${ }^{2}$, Marcin Rajewski ${ }^{1}$, \\ Tomasz Goździewicz ${ }^{1}$, Jana Skrzypczak ${ }^{1}$
}

${ }^{1}$ Department of Gynecology and Obstetrics, Division of Reproduction, Poznan University of Medical Sciences, Poland

${ }^{2}$ Gynecological and Obstetric Clinical Hospital in Poznan, Poland

\begin{abstract}
Factors controlling complement activation appear to exert a protective effect on pregnancy. This is particularly important in women with thrombophilia. The aim of this study was to determine the transcript and protein levels of complement decay-accelerating factor (DAF) and membrane cofactor protein (MCP) in the placentas of women with acquired and inherited thrombophilia. Also, we assessed immunohistochemistry staining of inhibitors of the complement cascade, DAF and MCP proteins, in the placentas of thrombophilic women. Placentas were collected from eight women with inherited thrombophilia and ten with acquired thrombophilia. The levels of DAF and MCP transcripts were evaluated by qPCR, the protein level was evaluated by Western blot. We observed a higher transcript $(\mathrm{p}<0.05)$ and protein $(\mathrm{p}<0.001)$ levels of DAF and MCP in the placentas of thrombophilic women than in the control group. DAF and MCP were localized on villous syncytiotrophoblast membranes, but the assessment of staining in all groups did not differ. The observed higher expression level of proteins that control activation of complement control proteins is only seemingly contradictory to the changes observed for example in the antiphospholipid syndrome. However, given the hitherto known biochemical changes associated with thrombophilia, a mechanism in which increased expression of DAF and MCP in the placentas is an effect of proinflammatory cytokines, which accompanies thrombophilia, is probable. (Folia Histochemica et Cytobiologica 2012, Vol. 50, No. 3, 460-467)
\end{abstract}

Key words: thrombophilia, complement, placenta, DAF, MCP

\section{Introduction}

Antiphospholipid Syndrome (APS) and coexisting thrombosis are being extensively studied as potential causes of pregnancy loss. The loss is more likely to occur in the first trimester of pregnancy as a result of impaired implantation, whereas intrauterine demise, placental abruption or preeclampsia are more frequent in later pregnancy [1]. Although the frequency of thrombophilia in pregnant women is lower than

Correspondence address: P.K. Wirstlein,

Department of Gynecology and Obstetrics,

Division of Reproduction,

Poznan University of Medical Sciences,

Polna Str. 33, 60-535 Poznan, Poland;

tel.: + 486184193 02, fax: + 486184196 25;

e-mail: abys@wp.pl previously believed, the association between various types of antiphospholipid (aPL) antibodies, factor V Leiden and prothrombin mutation is an evident cause of pregnancy complications [2].

Despite numerous studies, the mechanism of pregnancy loss in women with APS remains unclear. Antiphospholipid antibodies (aPL) in vitro inhibit the anticoagulant function of annexin $\mathrm{V}$, protein $\mathrm{C}$ and block fibrinolysis [3-6]. Besides activating the complement cascades, aPL (including $\beta_{2}$-glycoprotein 1 antibodies (anti- $\beta_{2}$ GP1) in the placenta) blocks the $\beta_{2} \mathrm{GP} 1$ protein as a ligand for receptors on epithelial and trophoblast cells surface, resulting in decreased hCG production and impaired expression of protein on cell surface (integrins, catherins). Anti- $\beta_{2}$ GP1 binding to trophoblast blocks its invasion inducing apoptosis. Anti- $\beta_{2}$ GP1 has been observed to affect trophoblast function, decreasing the expression 
of the growth factor: heparin binding EGF-like factor (HB-EGF) [7].

Complement activation due to aPL induces inflammatory reaction through proinflammatory cytokines including $\mathrm{TNF} \alpha$, thus impairing the balance between Th1/Th2 which is favorable in pregnancy [8]. The inflammatory reaction is also present in thrombophilia not related to APS. The key moment of this process is the activation of prothrombin into thrombin. Thrombin has a wide spectrum of action, fusing together proinflammatory and prothrombotic action and stimulating proinflammatory cytokine expression $[9,10]$.

Tissues including placenta possess the ability to inhibit complement cascade through the presentation of receptors for complement elements and membrane proteins degrading complement elements on the surface of cells. The complement decay-accelerating factor (DAF), also known as CD55, is among many well-studied complement control proteins. This membrane protein prevents the assembly of the $\mathrm{C} 3 \mathrm{bBb}$ complex, thus blocking the formation of the membrane attack. Another well-known complement inhibitory factor is the membrane cofactor protein (MCP), also known as CD46. MCP protects the host cells from damage as cofactor for inactivation of complement components $\mathrm{C} 3 \mathrm{~b}$ and $\mathrm{C} 4 \mathrm{~b}$ by serum factor $\mathrm{I}$.

It has been demonstrated that murine homozygotes without the Crry (Crry-/-) gene, encoding protein of analog action to human DAF and MCP, experience fetal absorption or growth impairment $[11,12]$.

The role of the complement controlling cascade factors in thrombophilia is poorly understood. Rai et al. (in 2006) showed that in non-conception cycle, the level of mRNA for DAF in the endometrium of APS patients is statistically lower compared to healthy women [13]. We can speculate that activation of complement cascade in APS may be associated with weakened expression of complement cascade inhibitors. Secondary complement activation takes place also in inherited thrombophilia through thrombin activation [10]. To date there have been very few papers concerning molecular changes in the placentas of women with thrombophilia. Nor is the status of complement controlling factors in thrombophilic patients well understood.

The aim of this study was to investigate whether in the placentas of thrombophilic women there is an altered expression of DAF and MCP proteins, and whether differences in the expression of these proteins are dependent on the form of thrombophilia.

\section{Material and methods}

Material. The study was conducted at the Division of Reproduction, Department of Obstetrics and Gynecology, Karol Marcinkowski Poznan University of Medical Sciences, Poland, in 2011.
We analyzed 19 placentas from 18 patients with inherited $(\mathrm{n}=8)$ and acquired $(\mathrm{n}=10)$ thrombophilia; 20 placentas from uneventful pregnancies which resulted in the delivery of a healthy infant constituted the control group $(n=20)$.

Patients with APS fulfilled at least one clinical and one laboratory criterion. Clinical criteria included: (1) three or more early miscarriages; or (2) two consecutive late miscarriages (beyond 12 weeks of gestation); or (3) intrauterine fetal death beyond ten weeks of gestation of unknown cause or delivery before 34 gestational weeks complicated by preeclampsia with placental insufficiency; or (4) thrombotic episodes.

Laboratory criteria included: (1) presence of lupus anticoagulant (LA); (2) moderate to high titers ( $\geq 20 \mathrm{GPL}$ or MPL) of anticardiolipin antibodies (aCL) or substantial titers (> 99 percentile) of anti- $\beta_{2}$ GP1. In molecular tests, eight women were diagnosed with inherited thrombophilia: factor V Leiden was found in five, hyperhomocysteinemia in two, and protein S deficiency in one patient. All pregnant patients diagnosed with thrombophilia were treated with either prophylactic or therapeutic doses of LMWH and/or aspirin, depending on their weight and thrombotic episodes. The clinical characteristics of the studied groups are shown in Table 1.

The study protocol was approved by the local ethical committee and the patients signed an informed consent form. All coagulation tests were performed at the Hemostasis Laboratory of Medical University Hematology Department in Poznan. The laboratory has a TUV Rheinland ISO9001 certificate.

Method. Directly after birth, a small part of the placenta was placed in RNAlater and frozen at minus $20^{\circ} \mathrm{C}$, for the assessment of transcript levels. Another part was frozen in liquid nitrogen (LN) until Western blot assessment was performed. The specimens included both maternal and fetal fragments, but the decidual and amniochorional membranes were removed. The entire placenta was fixed in buffered formalin for histopathological assessment and DAF and MCP immunolocalization.

RNA isolation and Real-time quantitative PCR (RQ-PCR) analysis of DAF and MCP transcript levels. Total RNA was isolated from $30 \mathrm{mg}$ of placenta by RNeasy Protect Mini Kit Qiagen (Hilden, Germany). RNA samples were treated with DNase I from DNase Set Qiagen (Hilden, Germany), quantified, and $1 \mu \mathrm{g}$ reverse-transcribed into cDNA, using QuantiTect Reverse Transcription Kit and random hexamer primers from Qiagen (Hilden, Germany). RQ-PCR was conducted in a Corbett Research Rotor-Gene 3000 thermocycler (Mortlake, Australia). Target cDNA was quantified using the relative quantification method employing a calibrator. The calibrator was prepared as a cDNA mix from all patients and control samples and consecutive dilutions were used to create a standard curve as provided in the 
Table 1. Clinical characteristics of women with thrombophilia and control group

\begin{tabular}{|c|c|c|c|}
\hline Characteristics & $\begin{array}{l}\text { Inherited thrombophilia } \\
\qquad(\mathbf{n}=\mathbf{8})\end{array}$ & $\begin{array}{l}\text { Acquired thrombophilia } \\
\qquad(\mathrm{n}=\mathbf{1 0})\end{array}$ & $\begin{array}{l}\text { Control group } \\
\quad(\mathbf{n}=20)\end{array}$ \\
\hline \multicolumn{4}{|l|}{ Maternal characteristics } \\
\hline Maternal age (mean \pm SD) & $30.2 \pm 3.16$ & $31.4 \pm 3.04$ & $29.7 \pm 3.97$ \\
\hline Gravity (mean \pm SD) & $1.75 \pm 1.39$ & $2.18 \pm 1.6$ & $1.1 \pm 0.31$ \\
\hline Parity $($ mean $\pm \mathrm{SD})$ & $0.38 \pm 0.52$ & $0.55 \pm 0.93$ & $1.1 \pm 0.31$ \\
\hline History of pregnancy failures* & & & - \\
\hline Early miscarriages & 2 & 4 & - \\
\hline Late miscarriages & 2 & 2 & - \\
\hline IUD & 3 & 4 & - \\
\hline Previous thrombotic episodes & 2 & 5 & - \\
\hline Laboratory criteria** & & & - \\
\hline $\mathrm{aCL}$ & - & 6 & \\
\hline anti- $\beta_{2}$ GPI & - & 1 & \\
\hline LA & - & 7 & \\
\hline F V Leiden & 5 & - & \\
\hline Hyperhomocysteinemia & 2 & - & \\
\hline Protein S deficiency & 1 & - & \\
\hline \multicolumn{4}{|c|}{ Characteristics of current pregnancy } \\
\hline Placental weight $[\mathrm{g}]$ & $562.86 \pm 164.39$ & $509.55 \pm 226.54$ & $530.0 \pm 96.57$ \\
\hline Fetal weight $[\mathrm{g}]$ & $2,893.75 \pm 1,285.22$ & $2,578.33 \pm 1,269.47$ & $3,351.0 \pm 398.15$ \\
\hline Gestation age (wk) & $36.75 \pm 3.99$ & $34.91 \pm 6.35$ & $39.2 \pm 1.32$ \\
\hline Vaginal delivery & 3 & 5 & 15 \\
\hline Cesarean section & 5 & 5 & 5 \\
\hline
\end{tabular}

*A number of women with thrombophilia showed more than one clinical criterion eligible for laboratory diagnostics;

** In two patients with acquired thrombophilia, we found concurrence of aCL and LA; in one concurrence of aCL, LA, and anti- $\beta_{2}$ GPI

Table 2. Sequence of primers used for qPCR

\begin{tabular}{|c|l|c|c|c|}
\hline Gene & Sequence (5'-3' direction)* & Position & $\begin{array}{c}\text { ENST number } \\
\text { www. ensembl.org }\end{array}$ & $\begin{array}{c}\text { Product } \\
\text { size (bp) }\end{array}$ \\
\hline \multirow{2}{*}{$D A F$} & GGTGCAACCATCTCCTTCTC & $\begin{array}{l}+805 ;+825 \\
+926 ;+946\end{array}$ & 00000196352 & 141 \\
& TGG TGG TGC TGG ACA ATA AA & $\begin{array}{l}+2,537 ;+2,558 \\
+2,615 ;+2,636\end{array}$ & 00000117335 & 99 \\
\hline \multirow{2}{*}{$A C P$} & TTGATTGTACCAAGGGATGGA & $\begin{array}{l}+450 ;+473 \\
+592 ;+616\end{array}$ & \multirow{2}{*}{00000331789} & 166 \\
& AACTGCTTGGCTAAGGGACTC & GCACCACACCTTCTACAATGAGC & & \multirow{2}{*}{} \\
& GGATAGCACAGCCTGGATAGCAAC & & & \\
\hline
\end{tabular}

*Source: http://medgen.ugent.be/rtprimerdb/ last accessed: 19 Aug. 2011

Relative Quantification Manual from Roche Diagnostics GmbH (Mannheim, Germany).

For amplification, $60 \mathrm{ng}$ of total cDNA solution was added to $20 \mu \mathrm{l}$ (total) of DyNAmo HS SYBR ${ }^{\circledR}$ Green qPCR Kit from Finnzymes (Espoo, Finland) and primers (Table 2). One RNA sample of each preparation was processed without the reverse transcription (RT)-reaction to provide a negative control in subsequent PCR. The quantity of in- vestigated transcripts in each sample was standardized by human $\beta$-actin (ACTB) transcript levels. The DAF and MCP mRNA levels were expressed as the multiplicity of these cDNA concentrations in the calibrator.

Protein isolation and Western blot analysis. The crushed in LN material was mixed with SB buffer (125 mM Tris$\mathrm{HCl}$ pH 6.8, 4\% SDS, $20 \%$ glycerol, $10 \%$ 2-mercaptoetha- 
nol) and total protein concentration was estimated using a commercial 2-D Quant kit (GE Healthcare). Samples containing $30 \mu \mathrm{g}$ of protein were separated by electrophoresis in a $4-12 \%$ SDS-polyacrylamide gel (Bis-Tris NuPAGE, Invitrogen). The separated proteins were transferred to a PVDF membrane and blocked with TBST (TBS plus $0.1 \%$ Tween-20) containing 4\% BSA.

Immunodetection was processed with the use of rabbit polyclonal antibody anti-DAF (H-319, $200 \mu \mathrm{g} / \mathrm{ml})$ and rabbit polyclonal antibody anti-MCP (H-294; $200 \mu \mathrm{g} / \mathrm{ml})$ in 1:200 concentrations (SantaCruz, USA). For ACTB detection, rabbit polyclonal anti-ACTB antibody was used in a $1: 400$ concentration $(\mathrm{N}-21 ; 100 \mu \mathrm{g} / \mathrm{ml})$, (SantaCruz, USA). Goat anti-rabbit, conjugated with Alexa Fluor 663 (Invitrogen, USA), was used as the secondary antibody. Bands were revealed using the Fuji FLA5100 (FujiFilm, Japan) scanner. The quantities of Western blot-detected DAF, MCP and $\beta$-actin proteins were determined based on the band optical density. The band densitometry readings were normalized to ACTB loading control to calculate the studied protein to $\beta$-actin optical density ratio. As reference for statistical calculations, the mean value of DAF and MCP in the control group was determined as 1.00 .

A

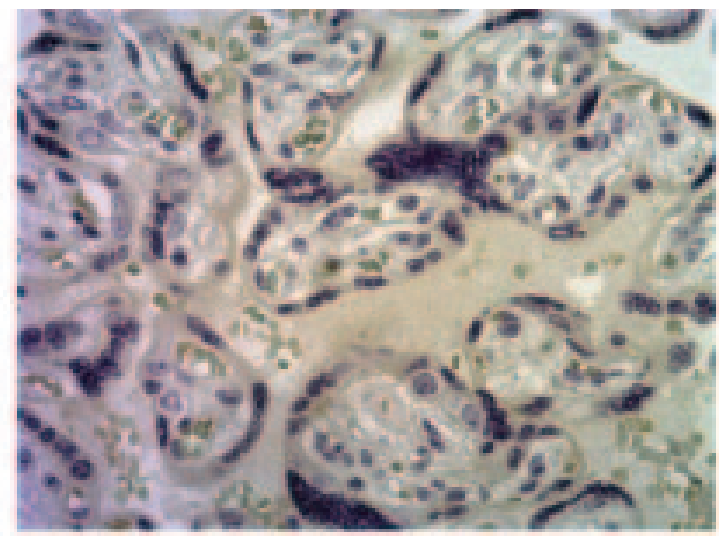

B

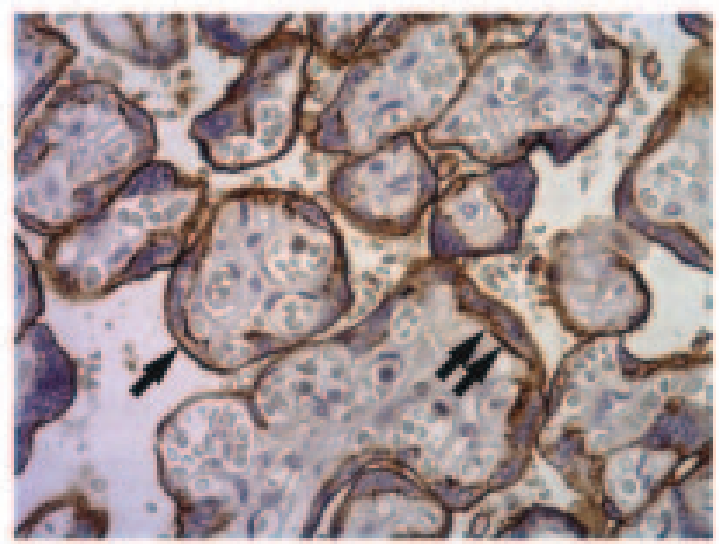

Immunohistochemistry. Samples from the placenta in unchanged regions were taken for immunohistochemistry. Specimens were preserved in buffered formalin $(\mathrm{pH} 7.4-$ -7.6), immersed in paraffin blocks and dissected into pieces up to 3-4 $\mu \mathrm{m}$ thick. A part of the sections was stained with hematoxylin and eosin $(\mathrm{H}+\mathrm{E})$ and neighboring sections underwent immunohistochemical staining to identify the presence of DAF and MCP antigen. Retrieval of antigens was processed in Retrieval Solution Buffer (DAKO, Denmark), $98.5^{\circ} \mathrm{C}$. To detect DAF and MCP, the same primary antibodies were used as in WB analysis in 1:100 dilution. Reaction with secondary antibodies and staining were performed with Dako REAL ${ }^{\mathrm{TM}}$ EnVision $^{\mathrm{TM}}$ Detection System Peroxidase/DAB, (DAKO, Denmark). With every series of staining, we performed a negative control without primary antibody.

Assessment of the immunohistochemical reaction was conducted by a pathologist in random order, with no knowledge of the study group affinity. The expression of DAF and MCP proteins was assessed with the use of a $0-3$ point scale $(0-$ no reaction (Figure $1 \mathrm{~A}) ; 1$ - weak reaction, discontinuous in both membranes in over $50 \%$ of the villi (Figure 1B); 2 - distinct reaction, continuous in one of the

C

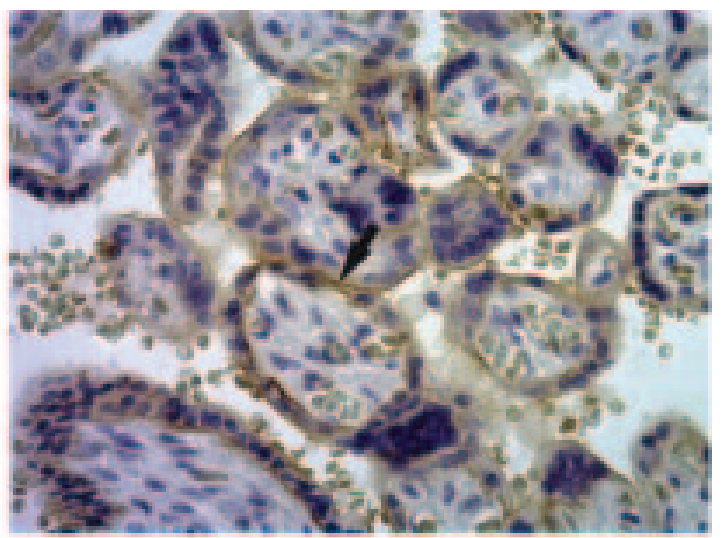

D

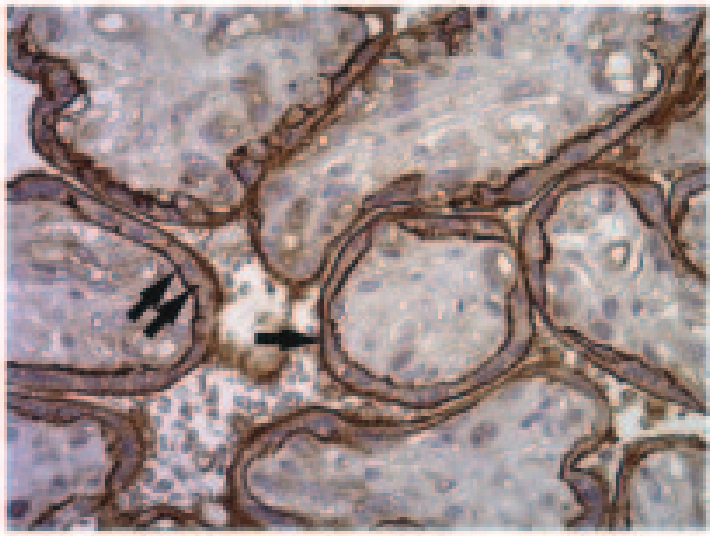

Figure 1. Immunohistochemical staining for DAF and MCP proteins, magnification $\times 400$. Lack of reaction (A). Subtle reaction in the outer membrane of syncytiotrophoblast (arrow) (B). Continuous reaction in the outer membrane of syncytiotrophoblast (arrow); discontinuous in the basement membrane (dual arrow) (C). Continuous reaction in both membranes of syncytiotrophoblast: superficial (arrow) and basal (dual arrow) (D) 
membranes in more than $50 \%$ of the villi (Figure 1C); $3-$ distinct reaction in both membranes in more than $50 \%$ of the villi (Figure 1D).

Statistical analysis. For statistical analysis, SigmaStat3.5 software was used. The analysis of the results was based on the Mann-Whitney Rank Sum test and Chi Square test for staining assessment. $\mathrm{p}<0.05$ was considered statistically significant.

\section{Results}

Levels of DAF and MCP transcript and protein in placentas of women with thrombophilia and controls

We used RQ-PCR and Western blot analysis to evaluate DAF and MCP transcript and protein levels, respectively, in the placentas of women diagnosed with thrombophilia and the controls. In women with acquired thrombophilia, we observed significantly higher levels $(\mathrm{p}<0.05)$ of DAF and MCP transcript in placentas with APS group compared to controls: 2.2 times for DAF and 2.29 times for MCP. In placentas from women with inherited thrombophilia, we also observed significantly higher levels $(p<0.05)$ of DAF and MCP transcript in placentas from the thrombosis group compared to controls: 4.52 times for DAF and 3.74 times for MCP (Table 3, Figures 2A, B).

We also found significantly higher levels of DAF and MCP protein $(\mathrm{p}<0.001)$ in placentas with acquired thrombophilia compared to controls: 3.26 times for DAF and 3.83 times for MCP. In the inherited thrombophilia group, we found 3.08 times higher level of DAF protein and 3.94 times higher level of MCP protein $(p<0.001)$ (Table 3, Figures $3 \mathrm{~A}, \mathrm{~B})$. Additionally, we found no difference in transcripts and proteins DAF and MCP between the inherited and acquired thrombophilia groups (data not shown).

\section{Immunohistochemistry staining}

Generally, a strong color reaction in the chorion syncytiotrophoblast membranes was observed for both tested proteins. Statistical analysis showed no statistically significant differences in the scale of staining for MCP and DAF. The results of histological evaluation of microscope slides, stained for the presence of proteins DAF and MCP, are shown in Table 4. In samples of the controls, stained with the anti-DAF antibody, a higher (although not statistically significant) percentage of staining assessed as 3 points, and a higher percentage of staining evaluated at 0 and 1 in the group of placentas from thrombophilic women, is worth noting.

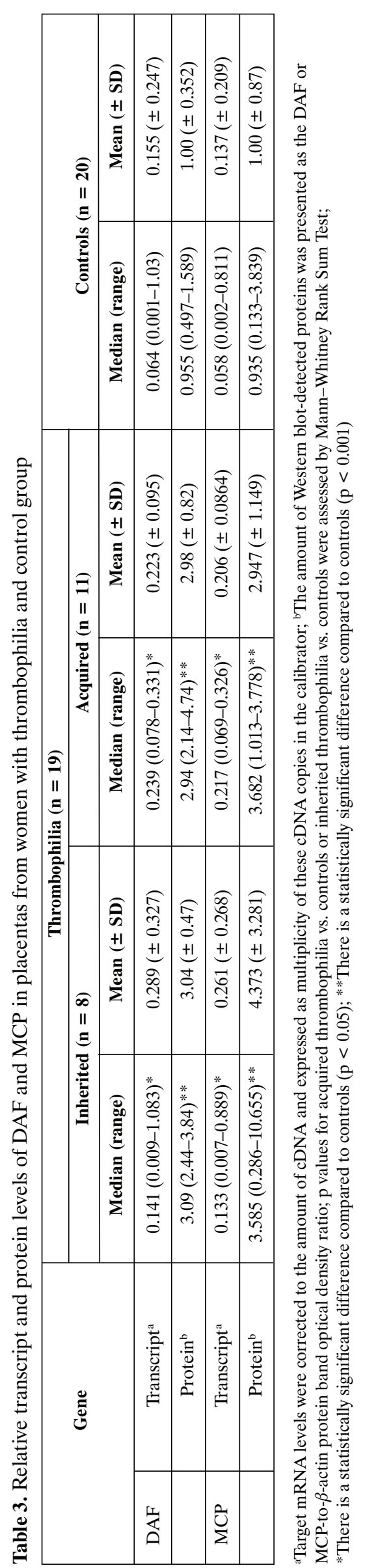

(C) Polish Society for Histochemistry and Cytochemistry 

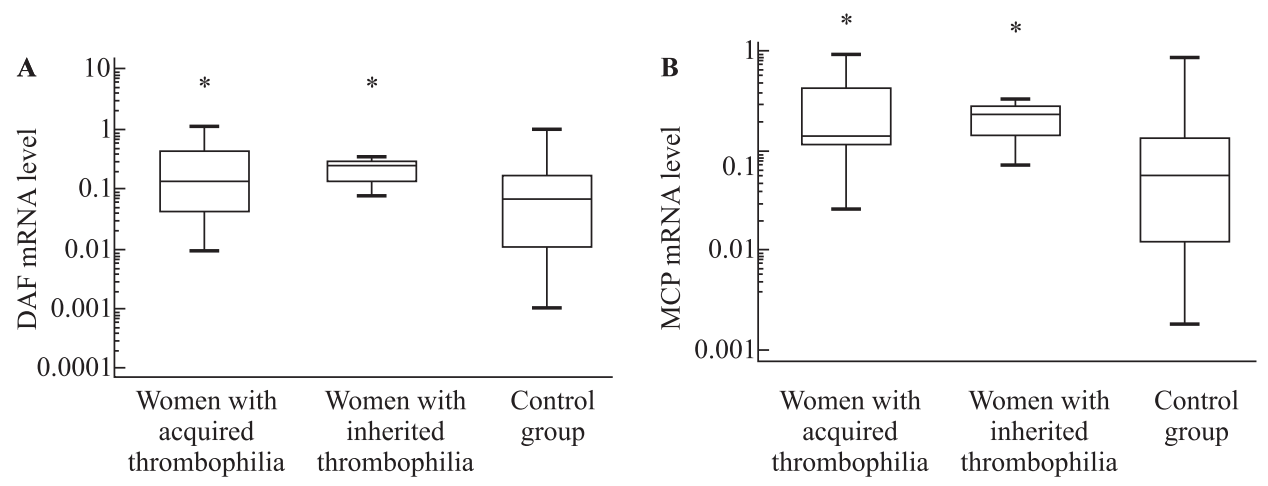

Figure 2. Relative transcript level of DAF $(\mathbf{A})$ and $\mathrm{MCP}(\mathbf{B})$ in placentas from women with acquired and inherited thrombophilia and control group. To normalize the quantity of transcripts in each sample, DAF and MCP mRNA levels were normalized to the amount of ACTB cDNA. The boxes and the middle lines correspond to the values from the lower to upper quartiles and medians, respectively. ${ }^{*} \mathrm{p}<0.05$
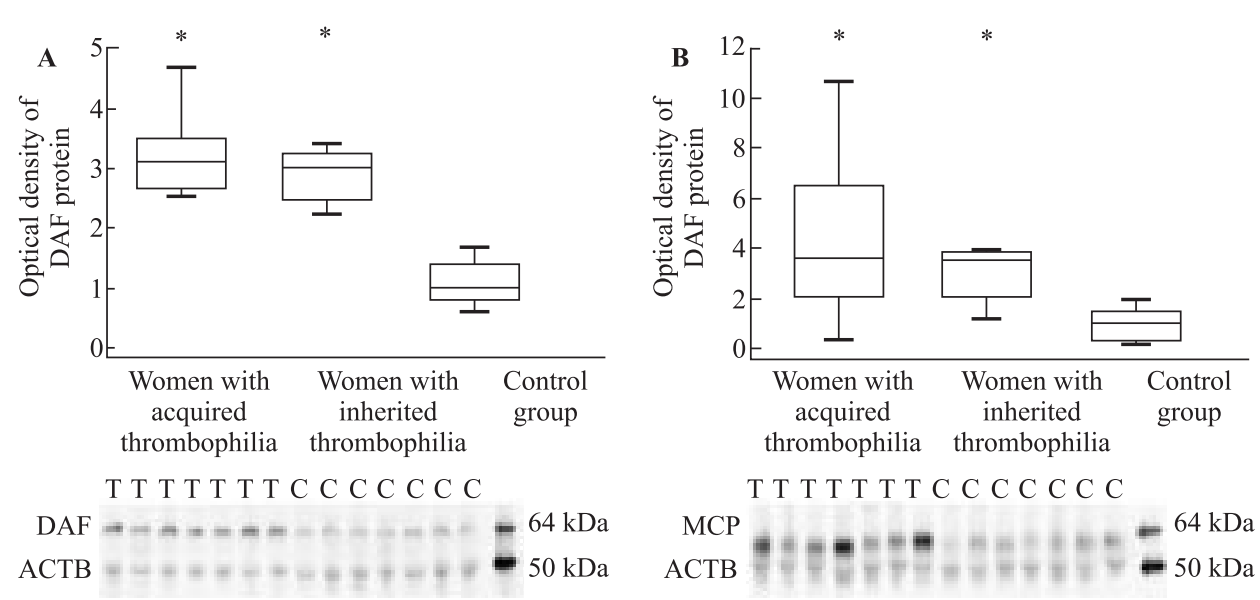

Figure 3. Protein levels for DAF (A) and MCP (B) and representative picture of Western blot analysis (below plots). The amount of Western blot-detected proteins was presented as the DAF/MCP-to- $\beta$-actin band optical density ratio. The boxes and the middle lines correspond to the values from the lower to upper quartiles and medians, respectively. *p $<0.001$

Table 4. Assessment of immunohistochemical staining for DAF and MCP proteins. The table shows the number of patients presenting successive staining steps $(0-3)$ of tissues for the presence of DAF and MCP

\begin{tabular}{|l|c|c|c|c|c|c|c|c|}
\hline $\begin{array}{l}\text { Degree of immunohisto- } \\
\text { chemical assessment }\end{array}$ & \multicolumn{4}{|c|}{ DAF $^{\mathrm{a}}$} & \multicolumn{4}{c|}{ MCP $^{\mathrm{b}}$} \\
\hline & $\mathbf{0}$ & $\mathbf{1}$ & $\mathbf{2}$ & $\mathbf{3}$ & $\mathbf{0}$ & $\mathbf{1}$ & $\mathbf{2}$ & $\mathbf{3}$ \\
\hline Acquired thrombophilia (\%) & 0 & $2(18 \%)$ & $9(82 \%)$ & 0 & 0 & 0 & $4(36 \%)$ & $7(64 \%)$ \\
\hline Inherited thrombophilia (\%) & $1(12.5 \%)$ & $3(37.5 \%)$ & $3(37.5 \%)$ & $1(12.5 \%)$ & $1(12.5 \%)$ & $1(12.5 \%)$ & $3(37.5 \%)$ & $3(37.5 \%)$ \\
\hline Control group (\%) & 0 & $1(5 \%)$ & $13(65 \%)$ & $6(30 \%)$ & 0 & 0 & $7(35 \%)$ & $13(65 \%)$ \\
\hline
\end{tabular}

${ }^{\mathrm{a}} \mathrm{Chi}^{2}, \mathrm{p}=0.099 ;{ }^{\mathrm{b}} \mathrm{Chi}^{2}, \mathrm{p}=0.201$

\section{Discussion}

Expression of complement activation proteins including MCP and DAF in trophoblast tissue is already observed in the first trimester of pregnancy. A strong expression of these proteins on trophoblast surface activates the mechanisms protecting the fe- tus from maternal immunological response [14, 15]. Hill et al. (1995) did not find any differences in DAF and MCP expression in the placentas of women with recurrent pregnancy losses in the first trimester compared to healthy fertile women [16]. Rai et al. (2006) proved a decreased transcript level of DAF in the endometrium of women with 
APS [13]. There is no data regarding the expression of complement activating proteins in mature placentas of thrombophilic women. Our results are in contrast with common scheme, where insufficient placental protection from DAF favors C3 convertase activation, resulting in inflammatory reaction in the placenta.

We observed significantly increased mRNA and protein expression of DAF and $\mathrm{MCP}$ in the placentas of women either with inherited thrombophilia or related to APS. It seems less likely that high expression of factors controlling the initial stage of complement cascade in APS women is primary. Otherwise we would have never observed the aPL related proinflammatory effect. aPL inhibiting the expression of HB-EGF would give an opposite effect to the one we found. The relationship between HB-EGF expression and DAF and MCP $[17,18]$ has been proved in both in vitro and in vivo studies.

An inhibiting function of aPL on HB-EGF secretion was confirmed in vitro only for the anti- $\beta_{2} \mathrm{GP} 1$ antibody. Simultaneously, it was confirmed that LMWH inhibits reaction of anti- $\beta_{2}$ GP1 with trophoblast [10].The influence of other types of antibodies on HB-EGF expression has not been determined.

In our study, in the group of women with APS, only one woman had an elevated titer of anti- $\beta_{2}$ GP1. We cannot exclude the possibility that the high expression of DAF and MCP in women with APS is indirectly a result of the treatment with therapeutic doses of LMWH or aspirin. In our study we found a statistically higher expression of DAF and MCP in both variants of thrombophilia.

There is no convincing data that could explain a primary high level of DAF and MCP in the placentas of women with inherited thrombophilia, where the inflammatory reaction mediator is likely the thrombin $[10,19]$. LMWH mechanism of action known from APS related thrombophilia is not applicable there. Considering the lack of difference in DAF and MCP level in both types of thrombophilia, it is less likely that LMWH has a stimulating effect on HB-EGF expression and indirectly on DAF and MCP.

Mason et al. (1999) observed a stimulating influence of TNF $\alpha$ in vitro on DAF expression in endothelial cells. There was no analog reaction as far as $\mathrm{MCP}$ was concerned [20]. Increased DAF expression found in our studies can be explained as a secondary response of placental tissue to inflammatory reaction and the presence of proinflammatory cytokine that can activate complement cascade in a secondary way $[9,10,20]$. Lack of similar observations in MCP in the referenced literature might be a result of the lim- ited possibilities of an in vitro model. It is very likely that a similar mechanism of secondary response to inflammation is associated with MCP expression in vivo. We support the theory that the DAF and MCP expression, as a secondary reaction to proinflammatory factors, is more likely. The presence of proinflammatory cytokines and thrombin is common for both variants of thrombophilia. Further studies are needed in order to explain this phenomenon.

Considering the limitation of quantitative analysis of protein expression in specimens stained immunohistochemically, only qualitative differences in placental distribution DAF and MCP were analyzed. There were no statistically significant differences in the evaluation of distribution of both proteins in the placenta, indicating no differences in cells mechanism responsible for the expression of analyzed placental factors.

To the best of our knowledge, this is the first publication analyzing the expression of DAF and MCP in the placentas of thrombophilic women. In this study we did not estimate the cytokine level. However, taking into consideration the abovementioned publication results of Mason et al., we can expect an elevated level of proinflammatory cytokines in the placentas of the thrombophilic group. Further studies are needed to elucidate whether proinflammatory cytokines in the placenta influence the expression of DAF and MCP expression and, if they do, what spectrum should be taken into consideration.

\section{Acknowledgements}

This study was supported by Ministry of Science and Higher Education grant NN407 056637 and UE grant $162 / E-392 / C D / D F S-4 / 2004$.

\section{References}

1. Ware Branch D, Eller AG. Antiphospholipid syndrome and thrombosis. Clin Obstet Gynecol. 2006;49:861-874.

2. Skrzypczak J, Jasiński P, Wirstlein P, Goździewicz T, Rajewski M. Histological changes in placenta and chorion of women with antiphospholipid syndrome and inherited thrombophilia. Ginekol Pol. 2011;82:652-663.

3. Rand JH, Wu XX, Quinn AS, Taatjes DJ. The annexin A5-mediated pathogenic mechanism in the antiphospholipid syndrome: role in pregnancy losses and thrombosis. $\mathrm{Lu}$ pus. 2010;19:460-469.

4. Rand JH, Wu XX, Guller S et al. Reduction of annexin-V (placental anticoagulant protein-I) on placental villi of women with antiphospholipid antibodies and recurrent spontaneous abortion. Am J Obstet Gynecol. 1994;171:1566-1572.

5. Krone KA, Allen KL, McCrae KR. Impaired fibrinolysis in the antiphospholipid syndrome. Curr Rheumatol Rep. 2010;12:53-57.

6. Meroni PL, Borghi MO, Raschi E, Tedesco F. Pathogenesis of antiphospholipid syndrome: understanding the antibodies. Nat Rev Rheumatol. 2011;7:330-339. 
7. Di Simone N, Marana R, Castellani R et al. Decreased expression of heparin-binding epidermal growth factor-like growth factor as a newly identified pathogenic mechanism of antiphospholipid-mediated defective placentation. Arthritis Rheum. 2010;62:1504-1512.

8. Meroni PL, Tedesco F, Locati M et al. Anti-phospholipid antibody mediated fetal loss: still an open question from a pathogenic point of view. Lupus. 2010;19:453-456.

9. Borissoff JI, Spronk HM, Heeneman S, ten Cate H. Is thrombin a key player in the 'coagulation-atherogenesis' maze? Cardiovasc Res. 2009;82:392-403.

10. Hamad OA, Ekdahl KN, Nilsson PH et al. Complement activation triggered by chondroitin sulfate released by thrombin receptor-activated platelets.J Thromb Haemost. 2008;6:1413-1421.

11. Zipfel PF, Skerka C. Complement regulators and inhibitory proteins. Nat Rev Immunol. 2009;9:729-740.

12. Xu C, Mao D, Holers VM, Palanca B, Cheng AM, Molina H. A critical role for murine complement regulator crry in fetomaternal tolerance. Science. 2000;287:498-501.

13. Francis J, Rai R, Sebire NJ et al. Impaired expression of endometrial differentiation markers and complement regulatory proteins in patients with recurrent pregnancy loss associated with antiphospholipid syndrome. Mol Hum Reprod. 2006;12:435-442.

14. Tedesco F, Narchi G, Radillo O, Meri S, Ferrone S, Betterle C Susceptibility of human trophoblast to killing by human com- plement and the role of the complement regulatory proteins. J Immunol. 1993;151:1562-1570.

15. Salmon JE, Girardi G. Antiphospholipid antibodies and pregnancy loss: adisorder of inflammation. J Reprod Immunol. 2008;77:51-56.

16. Hill JA, Melling GC, Johnson PM. Immunohistochemical studies of human uteroplacental tissues from first-trimester spontaneous abortion. Am J Obstet Gynecol. 1995;173:90-96.

17. Young SL, Lessey BA, Fritz MA et al. In vivo and in vitro evidence suggest that HB-EGF regulates endometrial expression of human decay-accelerating factor. J Clin Endocrinol Metab. 2002;87:1368-1375.

18. Lessey BA, Gui Y, Apparao KB, Young SL, Mulholland J. Regulated expression of heparin-binding EGF-like growth factor (HB-EGF) in the human endometrium: a potential paracrine role during implantation. Mol Reprod Dev. 2002;62:446-455.

19. O'Neil KM. Role of complement in antiphospholipid antibody-mediated thrombosis. Curr Rheumatol Rep. 2007;9:205$-211$.

20. Mason JC, Yarwood H, Sugars K, Morgan BP, Davies KA, Haskard DO. Induction of decay-accelerating factor by cytokines or the membrane-attack complex protects vascular endothelial cells against complement deposition. Blood. 1999;94:1673-1682. 